\title{
Analytical BPS Maxwell-Higgs Vortices
}

\author{
R. Casana, ${ }^{1}$ M. M. Ferreira Jr., ${ }^{1}$ E. da Hora, ${ }^{1,2}$ and C. dos Santos ${ }^{3}$ \\ ${ }^{1}$ Departamento de Física, Universidade Federal do Maranhão, 65080-805 São Luís, MA, Brazil \\ ${ }^{2}$ Coordenadoria do Curso Interdisciplinar em Ciência e Tecnologia, Universidade Federal do Maranhão, \\ 65080-805 São Luís, MA, Brazil \\ ${ }^{3}$ Centro de Física e Departamento de Física e Astronomia, Faculdade de Ciências da Universidade do Porto, 4169-007 Porto, Portugal \\ Correspondence should be addressed to E. da Hora; edahora.ufma@gmail.com
}

Received 14 January 2014; Revised 29 May 2014; Accepted 30 May 2014; Published 2 July 2014

Academic Editor: Kingman Cheung

Copyright (C) 2014 R. Casana et al. This is an open access article distributed under the Creative Commons Attribution License, which permits unrestricted use, distribution, and reproduction in any medium, provided the original work is properly cited. The publication of this article was funded by SCOAP $^{3}$.

\begin{abstract}
We have established a prescription for the calculation of analytical vortex solutions in the context of generalized Maxwell-Higgs models whose overall dynamics is controlled by two positive functions of the scalar field, namely, $f(|\phi|)$ and $w(|\phi|)$. We have also determined a natural constraint between these functions and the Higgs potential $U(|\phi|)$, allowing the existence of axially symmetric Bogomol'nyi-Prasad-Sommerfield (BPS) solutions possessing finite energy. Furthermore, when the generalizing functions are chosen suitably, the nonstandard BPS equations can be solved exactly. We have studied some examples, comparing them with the usual Abrikosov-Nielsen-Olesen (ANO) solution. The overall conclusion is that the analytical self-dual vortices are well-behaved in all relevant sectors, strongly supporting the consistency of the respective generalized models. In particular, our results mimic well-known properties of the usual (numerical) configurations, as localized energy density, while contributing to the understanding of topological solitons and their description by means of analytical methods.
\end{abstract}

\section{Introduction}

In the context of classical field theories, structures possessing topologically nontrivial profiles are usually described as the static solutions of the Euler-Lagrange equations in the presence of finite energy boundary conditions [1]. In some special cases, by requiring the minimization of the corresponding energy functional, such structures can also be described via a set of first-order differential equations also known as BPS equations [2,3], which provide genuine solutions of the Euler-Lagrange ones.

The kink is a one-dimensional topological object arising within the simplest field model containing only a single real scalar field [4]. Regarding higher-dimensional scenarios, the vortex stands for a planar configuration solving some radially symmetric Abelian-Higgs models $[5,6]$, whilst the magnetic monopole is a three-dimensional spherically symmetric object appearing in the non-Abelian-Higgs case $[7,8]$. All these solutions possess the minimum energy possible, being stable against decaying into their respective mesons.
Moreover, it is well known that, in order to give rise to topological fields, the corresponding model must allow for the spontaneous symmetry breaking mechanism, with its potential term presenting at least two asymmetric vacua, since topological defects are known to be formed during symmetry breaking phase transitions.

During the last years, a new kind of topologically nontrivial objects has been intensively studied in connection with field models endowed with noncanonical kinetic terms which change the dynamics of the overall system in a nonusual way. It is worthwhile to point out that the motivation regarding exotic dynamics arises in a rather natural way in the context of the string theories. In particular, given some special constraints, field models possessing nonusual dynamics also support minimum energy solutions; see, for instance, [9-17]. Moreover, except for their nontrivial nature, these solutions behave in the same general way as their standard counterparts do. On the other hand, exotic kinetic terms also induce slightly variations on the shape of the corresponding field solutions, changing their amplitudes and/or characteristic 
lengths. Nonstandard field models are defined by introducing generalizing functions on usual field models. Detailed investigations regarding topological defects in the context of these models are found in [18-35]. Many authors have also studied interesting applications of these new solutions within several different scenarios, specially involving the accelerated inflationary phase of the universe [36] via the so-called $k$ essence models [37], strong gravitational waves [38], tachyon matter [39], dark matter [40], and others [41-43].

Besides the variations on the defect amplitudes and characteristic lengths, these generalizing functions also provide new features for some models, as, for example, self-dual analytical solutions which certainly enriches our understanding about integrable systems. Recently, self-dual analytical monopoles were achieved in [44] in the context of some generalized Yang-Mill models [45]. These new analytical solutions, unattainable in the absence of the modifying functions, were divided into two different classes according to their capability of recovering (or not) the standard 't Hooft-Polyakov result. By following the purpose of achieving analytical solutions for topological defects, the present paper aims at investigating the existence of analytical BPS vortex solutions within the framework of the generalized MaxwellHiggs model [46].

The letter is organized as follows. In Section 2, we review some important details regarding the generalized MaxwellHiggs scenario. Section 3 is devoted to describing the prescription implemented to find the analytical BPS solutions of the generalized model. The consistence of our approach is verified by investigating some explicit examples. In the sequel, the new solutions are compared to the ANO profiles, allowing the identification of the main properties acquired by them. Finally, in Section 4, we present our final remarks and conclusions.

\section{The Nonstandard Model}

We begin by reviewing the $(1+2)$-dimensional generalized Maxwell-Higgs model introduced in [46], whose Lagrangian density is

$$
\mathscr{L}=-\frac{f^{2}(|\phi|)}{4} F_{\mu \nu} F^{\mu \nu}+w(|\phi|)\left|D_{\mu} \phi\right|^{2}-U(|\phi|),
$$

where $F_{\mu \nu}=\partial_{\mu} A_{\nu}-\partial_{\nu} A_{\mu}$ is the usual field strength tensor and $D_{\mu} \phi=\partial_{\mu} \phi-i e A_{\mu} \phi$ stands for the covariant derivative. Moreover, $f^{2}(|\phi|)$ and $w(|\phi|)$ are positive functions which change the dynamics of the overall model, being called dielectric functions because they mimic some effective electrodynamics in continuous media, as already mentioned in the literature. So far, the possibility of such generalizing functions to provide exactly solvable models for vortex configurations was not explored, being the main motivation of this paper. Here, for simplicity, all fields, coordinates, and parameters are supposed to be dimensionless.

The corresponding Euler-Lagrange equation for the gauge field is

$$
\partial_{\nu}\left(f^{2} F^{\nu \mu}\right)=J^{\mu}
$$

where $J^{\mu}=\operatorname{iew}\left(\phi \overline{D^{\mu} \phi}-\bar{\phi} D^{\mu} \phi\right)$ is the generalized current vector, which is also conserved $\left(\partial_{\mu} J^{\mu}=0\right)$. The stationary Gauss law then reads

$$
\partial_{k}\left(f^{2} \partial_{k} A_{0}\right)=2 e^{2} w A_{0}|\phi|^{2}
$$

being trivially verified by $A_{0}=0$, revealing that the static configurations of the generalized model (1) generate no electric field.

Stationary Ampère's law can be written as (already using $\left.A_{0}=0\right)$

$$
\epsilon_{i k} \partial_{k}\left(f^{2} B\right)=J_{i}
$$

whilst the equation controlling the Higgs field is

$$
w D_{k} D_{k} \phi+\left(\partial_{k} w\right) D_{k} \phi-\left|D_{k} \phi\right|^{2} \frac{\partial w}{\partial \bar{\phi}}=B^{2} f \frac{\partial f}{\partial \bar{\phi}}+\frac{\partial U}{\partial \bar{\phi}}
$$

Here, $B=\epsilon_{j k} \partial_{j} A_{k}$ represents the magnetic field.

In order to obtain the first-order self-dual equations of the model (1), we start from the expression for the generalized total energy; that is,

$$
E=\int\left(\frac{1}{2} f^{2} B^{2}+w\left|D_{k} \phi\right|^{2}+U\right) d^{2} x,
$$

which can also be written in the form

$$
\begin{aligned}
E=\int\left(\frac{1}{2}(\right. & f B \mp \sqrt{2 U})^{2}+w\left|D_{ \pm} \phi\right|^{2} \pm B(f \sqrt{2 U}) \\
& \left. \pm i w \epsilon_{i k}\left(\partial_{i} \phi\right)\left(\partial_{k} \bar{\phi}\right) \mp e w \epsilon_{i k} A_{k} \partial_{i}|\phi|^{2}\right) d^{2} x .
\end{aligned}
$$

The energy is minimized by imposing

$$
D_{ \pm} \phi=0, \quad B= \pm \frac{\sqrt{2 U}}{f},
$$

which are the generalized self-dual BPS equations. Considering (8), the BPS energy is then reduced to

$$
\begin{aligned}
E_{\mathrm{BPS}}= \pm \int\left(\epsilon_{i k} \partial_{i} A_{k}(f \sqrt{2 U})\right. & \\
& \left.-e w \epsilon_{i k} A_{k} \partial_{i}|\phi|^{2}+i w \epsilon_{i k}\left(\partial_{i} \phi\right)\left(\partial_{k} \bar{\phi}\right)\right) d^{2} x,
\end{aligned}
$$

and static Ampère's law is rewritten as

$$
\partial_{k}(f \sqrt{2 U})=-e w \partial_{k}|\phi|^{2}
$$

With it, the BPS energy becomes

$$
E_{\mathrm{BPS}}= \pm \int\left(\epsilon_{i k} \partial_{i}\left(A_{k} f \sqrt{2 U}\right)+i w \epsilon_{i k}\left(\partial_{i} \phi\right)\left(\partial_{k} \bar{\phi}\right)\right) d^{2} x
$$

The point to be clarified here is that the integrand in (11) can be reduced to a total derivative only when considering axially symmetric configurations. In this context, (10) stands for 
the key condition for attaining self-duality in this generalized Maxwell-Higgs theory.

Hence, from now on, the fields are supposed to be described by the usual axially symmetric vortex Ansatz:

$$
\phi(r, \theta)=v g(r) e^{i n \theta}, \quad \mathbf{A}(r, \theta)=-\frac{\hat{\theta}}{e r}(a(r)-n),
$$

where $n= \pm 1, \pm 2, \pm 3, \ldots$ stands for the vorticity of the resulting configuration, and the magnetic field is

$$
B(r)=-\frac{1}{e r} \frac{d a}{d r}
$$

The profile functions $g(r)$ and $a(r)$ are constrained to behave according to the standard boundary conditions

$$
\begin{array}{ll}
g(0)=0, & g(\infty)=1, \\
a(0)=n, & a(\infty)=0,
\end{array}
$$

giving rise to regular solutions possessing finite energy, as desired.

Now, we come back to (11) defining it in terms of the energy density $\varepsilon_{\mathrm{bps}}$ related to the BPS solutions as

$$
E_{\mathrm{bps}}=\int \varepsilon_{\mathrm{bps}} d^{2} x
$$

where

$$
\varepsilon_{\mathrm{bps}}=\mp \frac{1}{e r} \frac{d H}{d r},
$$

with the auxiliary function $H(r)$ being given by

$$
H(r) \equiv a f \sqrt{2 U}
$$

This function is finite at origin, $H(0)=H_{0}$, and fulfills $H(\infty)=0$. Observing these boundary conditions for $H(r)$, the resulting total energy (16) is

$$
E_{\mathrm{bps}}=\frac{2 \pi}{e}\left|H_{0}\right|
$$

One also remarks that $H_{0}$ is proportional to $n$, the winding number characterizing the vortex solution.

In terms of $g(r)$ and $a(r)$, the BPS equations read

$$
\begin{aligned}
& \frac{d g}{d r}= \pm \frac{a g}{r}, \\
& B= \pm \frac{\sqrt{2 U}}{f}
\end{aligned}
$$

which solve the Euler-Lagrange equations of motion. In order to perform such verification explicitly, we first write Ampère's law (4) in its axially symmetric form

$$
\frac{d}{d r}\left(f^{2} B\right)=-2 e v^{2} w \frac{g^{2} a}{r}
$$

which becomes

$$
\frac{d}{d r}(f \sqrt{2 U})=-2 e v^{2} w g \frac{d g}{d r}
$$

when (20) and (21) are used. The form (23) recovers the very same condition (10) that assures the self-duality of the overall model, revealing the consistence of the self-dual equations with Ampère's law. In addition, one can express (5) for the Higgs field in terms of $g(r)$ and $a(r)$; that is,

$$
\begin{gathered}
\frac{d^{2} g}{d r^{2}}+\frac{1}{r} \frac{d g}{d r}-\frac{a^{2} g}{r^{2}}+\frac{1}{2 w}\left(\left(\frac{d g}{d r}\right)^{2}-\frac{a^{2} g^{2}}{r^{2}}\right) \frac{d w}{d g} \\
=\frac{1}{2 w v^{2}}\left(B^{2} f \frac{d f}{d g}+\frac{d U}{d g}\right) .
\end{gathered}
$$

It simply provides

$$
U=\frac{1}{2} f^{2} B^{2}
$$

when saturated by the self-dual equations, which coincides with (21). In this way, we have explicitly shown that the selfdual equations solve the stationary Euler-Lagrange equations of motion.

Due to the arbitrariness of $f(g)$ and $w(g)$, the search for solutions to the axially symmetric Euler-Lagrange equations can be a quite hard task, even in the presence of the suitable boundary conditions (14) and (15). A way to circumvent this point is focusing the attention on the self-dual equations (20) and (21). However, it is worthwhile to reinforce that such equations only hold when the model is constrained by condition (10), also expressed as

$$
\frac{d}{d g}(f \sqrt{2 U})=-2 e v^{2} w g
$$

We can summarize in the following way: given a set of functions $f, w$, and $U$ satisfying (26), regular solutions $g(r)$ and $a(r)$ can be found by solving (20) and (21) using the boundary conditions (14) and (15). The resulting configurations stand for topological vortices possessing finite energy given by (19), which remains proportional to the magnetic flux $\Phi_{B}=2 \pi n / e$. The proportionality constant is finite and related to the value of $f \sqrt{2 U}$ near the origin. Moreover, it is worthwhile to point out that

$$
\varepsilon_{\mathrm{bps}}=2 U+2 v^{2} w\left(\frac{a g}{r}\right)^{2}
$$

is the BPS energy density (17), which becomes positive whenever (26) ensures a positive $w$ (for a given pair of functions $U$ and $f$ conveniently chosen).

The next section introduces some effective MaxwellHiggs models for which the BPS equations (20) and (21) can be solved analytically (instead of numerically, as usually done). The analytical profiles representing $g(r), a(r), B(r)$, and $\varepsilon_{\text {bps }}$ (17) are depicted and compared with the usual (numerical) ANO solution. Furthermore, the main features of the new analytical vortices are highlighted. 


\section{Analytical BPS Vortices}

Now, we present the main goal of this work by introducing generalized Maxwell-Higgs models for which the BPS equations (20) and (21) can be solved analytically according the finite energy boundary conditions (14) and (15). Here, for simplicity, we only consider those configurations for which the winding number is equal to the unity $(n=1)$, although it is also possible to find solutions with higher vorticity, as it will be explained below.

Along this section, we work with the upper signs in (20), (21), and (17) only. Also, for simplicity, we set $e=v=1$. Our prescription to find analytical self-dual vortices can be described as follows. Firstly, we choose the potential $U(g)$ supporting the spontaneous symmetry breaking of the $U(1)$ local gauge symmetry inherent to model (1). In the sequel, we choose an analytical function $g(r)$ satisfying the boundary conditions (14). Then, we use $g(r)$ to solve (20), which allows obtaining the corresponding profile for $a(r)$ fulfilling the boundary conditions (15). In the end, we use (21) to evaluate the expression for the generalizing function $f$, writing it as a function of the radial variable $r$ (i.e., regarding the analytical models as effective ones).

A general observation about the kinetic functions $f^{2}(|\phi|)$ and $w(|\phi|)$ is that they are presented as functions of the radial variable $r$, not of the field variable $g$, that is, $|\phi|$. Expressing $f$ and $w$ in terms of $g$ gives very long expressions, when possible. In general, it becomes a very difficult task.

The analytical profiles here obtained provide a set of selfdual vortices possessing finite total energy given by (19). It is also worthwhile to remember that the corresponding $f$ 's and $w$ 's are positive, as required. These new solutions are shown in Figures 1, 2, 3, and 4, from which we highlight their main features.

3.1. $|\phi|^{4}$-Models. We first investigate some models defined by the usual fourth-order Higgs potential

$$
U(g)=\frac{1}{2}\left(1-g^{2}\right)^{2}
$$

where the coupling constant for the scalar-matter selfinteraction was supposed to be equal to the unity, for simplicity.

The $|\phi|^{4}$-models here presented possess a generalizing function $f(r)$ finite at the boundaries (i.e., for $r=0$ and asymptotically). In this case, the BPS equations (20) and (21) reduce to

$$
\begin{gathered}
\frac{d g}{d r}=\frac{a g}{r} \\
\frac{1}{r} \frac{d a}{d r}=\frac{g^{2}-1}{f} .
\end{gathered}
$$

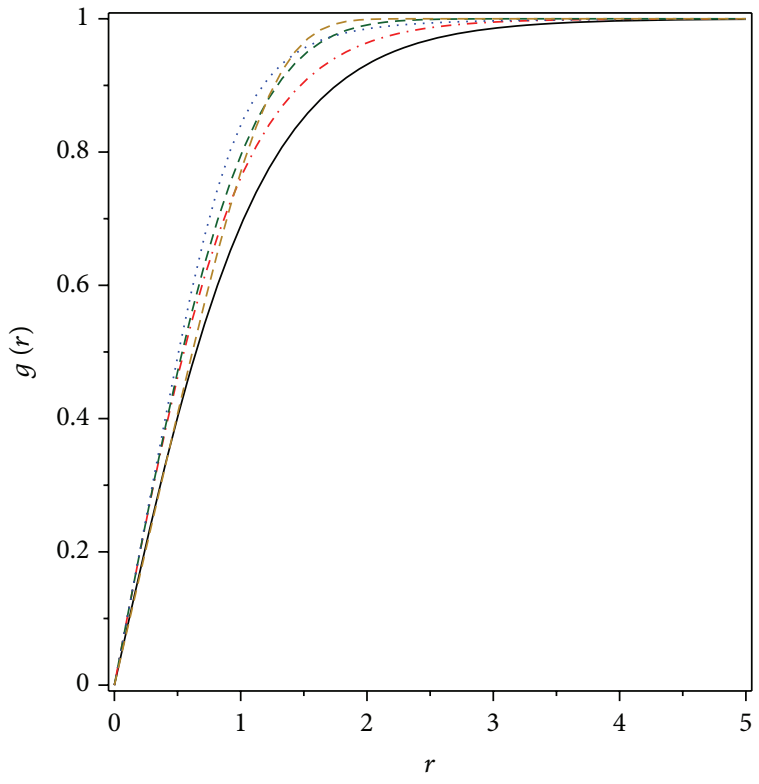

FIGURE 1: Solutions to $g(r)$ given by (32) (dash-dotted red line), (37) (dashed green line), (45) (dotted blue line), and (50) (long-dashed gold line). Here, the solid black line is the standard (numerical) $n=1$ ANO solution.

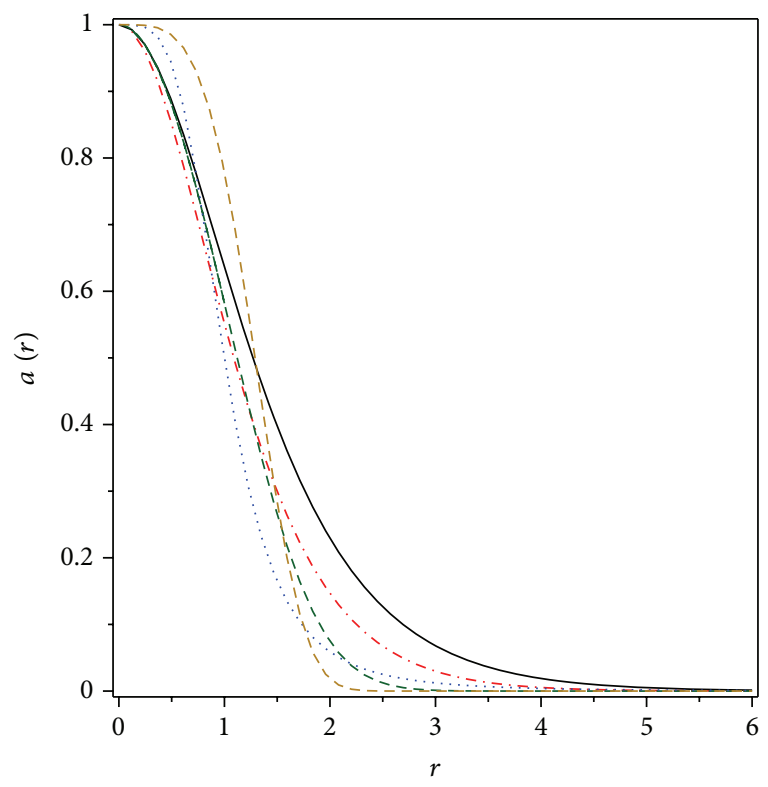

FIgURE 2: Solutions to $a(r)$. Conventions as in Figure 1.

In particular, one clearly sees that $f=1$ leads us back to the standard case

$$
\begin{gathered}
\frac{d g}{d r}=\frac{a g}{r}, \\
\frac{1}{r} \frac{d a}{d r}=g^{2}-1,
\end{gathered}
$$

which yields the well-known Abrikosov-Nielsen-Olesen numerical vortices $[5,6]$. Moreover, families containing 


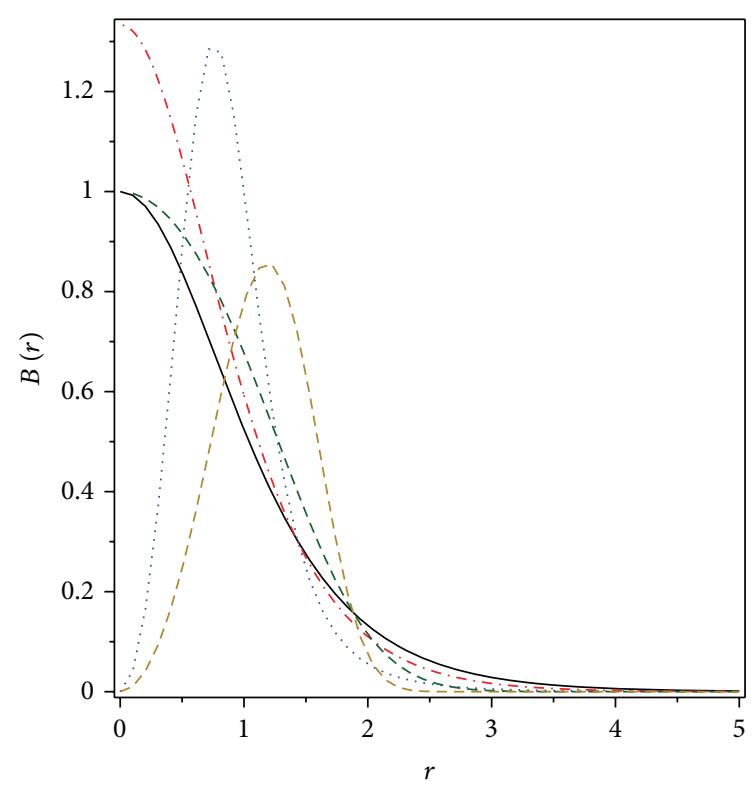

FIgUre 3: Solutions to $B(r)$. Conventions as in Figure 1.

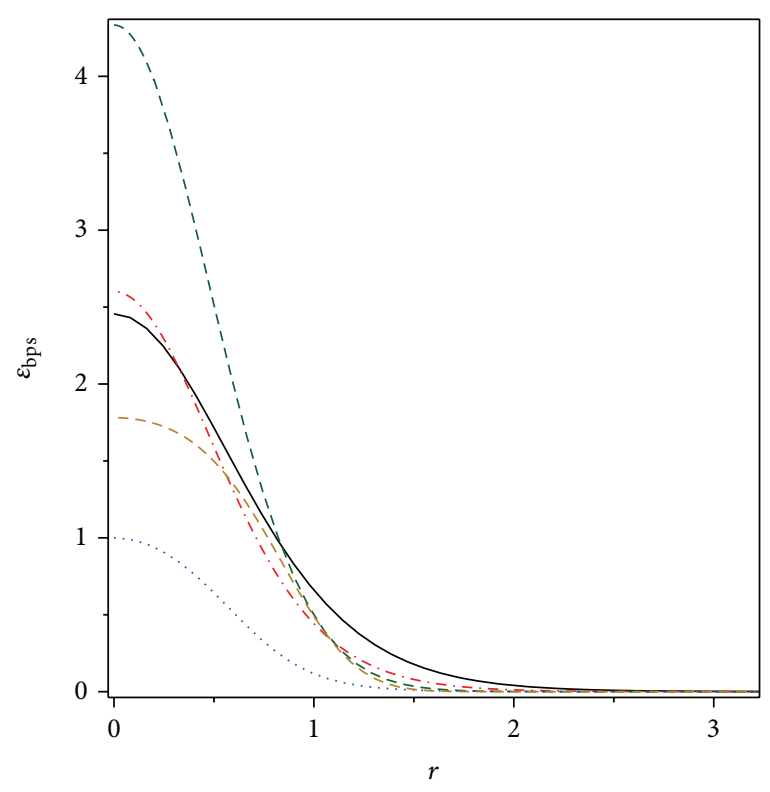

Figure 4: Solutions to $\varepsilon_{\mathrm{bps}}$. Conventions as in Figure 1.

nontrivial numerical solutions of the same kind were also studied in [46].

We now proceed looking for analytical solutions of (29) and (30). In this sense, by following our prescription, the first model we introduce is defined by the BPS solution

$$
g(r)=\tanh (r),
$$

which trivially obeys (14). Replacing it in (29), one achieves the gauge field profile

$$
a(r)=\frac{2 r}{\sinh (2 r)},
$$

satisfying the boundary conditions (15), with $n=1$. Now, by using (32) and (33) in (30), one gets the fact that the corresponding function $f(r)$ reads as

$$
f(r)=\frac{r(1-\cosh (2 r))}{\sinh (2 r)-2 r \cosh (2 r)},
$$

being a smooth and positive function with values $f(0)=3 / 4$ and $f(\infty)=1 / 2$. Finally, we obtain the auxiliary function $H$ by substituting (28), (33), and (34) into (18). The resulting expression is

$$
H(r)=\frac{r^{2} \sinh (r)}{\left((1 / 2) \sinh (2 r)-2 r \cosh ^{2}(r)+r\right) \cosh ^{3}(r)},
$$

a smooth function for which the values at the boundaries read as $H(0)=-3 / 4$ and $H(\infty)=0$, as desired. Finally, the magnetic field associated with (33) is given by

$$
B(r)=\frac{2(2 r \cosh (2 r)-\sinh (2 r))}{r \sinh ^{2}(2 r)},
$$

whose profile is a lump centered at the origin; see the dashdotted red line in Figure 3. Therefore, the solutions (32) and (33) represent analytical BPS Maxwell-Higgs vortices possessing total energy equal to $E_{\mathrm{bps}}=3 \pi / 2$, according to (19).

The second model, inherent to the $|\phi|^{4}$-potential, is defined by the BPS solution

$$
g(r)=\sqrt{1-e^{-r^{2}}}
$$

which also fulfills the conditions (14). From (29), we attain the gauge field profile

$$
a(r)=\frac{r^{2}}{e^{r^{2}}-1},
$$

satisfying the boundary conditions (15) (also with $n=1$ ). Then, by combining (37) and (38) in (30), the following generalizing function is achieved:

$$
f(r)=\frac{\left(e^{-r^{2}}-1\right)^{2}}{2\left(e^{-r^{2}}+r^{2}-1\right)} .
$$

This is a positive and finite function whose values at the boundaries are $f(r=0)=1$ and $f(r=\infty)=0$. Moreover, the corresponding $H(r)$ is given by

$$
H(r)=\frac{r^{2} e^{-2 r^{2}}\left(e^{-r^{2}}-1\right)}{2\left(e^{-r^{2}}+r^{2}-1\right)},
$$

providing $H(0)=-1$ and $H(\infty)=0$, as required. The magnetic field,

$$
B(r)=\frac{2 e^{-r^{2}}\left(e^{-r^{2}}+r^{2}-1\right)}{\left(e^{-r^{2}}-1\right)^{2}},
$$


also is a lump centered at the origin; see the dashed green line in Figure 3. This way, the profiles equations (37) and (38) describe analytical self-dual vortices whose BPS total energy is $E_{\mathrm{bps}}=2 \pi$. In particular, it means that these analytical solutions saturate the very same Bogomol'nyi bound fulfilled by the usual (numerical) $n=1 \mathrm{ANO}$ vortex.

3.2. $|\phi|^{6}$-Models. As was shown in [46], the generalized model allows finding BPS vortices even in the presence of a higher-order potential describing the scalar-matter selfinteraction. In this sense, we now go further by introducing analytical self-dual vortices arising in the presence of a sixthorder potential, defined by

$$
U(g)=\frac{1}{2} g^{2}\left(1-g^{2}\right)^{2}
$$

The vacuum manifold of the corresponding $|\phi|^{6}$-model is represented by a dot surrounded by a circle, with the dot standing for a symmetric vacuum. As a consequence, under a suitable choice of the boundary conditions to be satisfied by the profile functions $g(r)$ and $a(r)$, the model also supports nontopological self-dual structures possessing finite energy. Indeed, some of us have already obtained such objects, with results being forthcoming reported [47]. It is worthwhile to point out that nontopological vortices do not occur in the $|\phi|^{4}$-model (28) because it has no a symmetric vacuum (i.e., its vacuum manifold is a circle).

On the other hand, it is well known that the $|\phi|^{6}$-potential (42) ensures the self-duality of the usual Chern-SimonsHiggs (CSH) model, whose topological vortices possess both electric and magnetic fields. Despite the fact that our generalized $|\phi|^{6}$-Maxwell-Higgs model supports only noncharged self-dual solutions, they are expected to behave in the same general way the CSH ones do. Some numerical self-dual $|\phi|^{6}$-Maxwell-Higgs models were already investigated in [46]. Here, for completeness, we consider only the analytical solutions for it.

Returning to our prescription, under the $|\phi|^{6}$-potential (42), the BPS equations (20) and (21) can be written as

$$
\begin{gathered}
\frac{d g}{d r}=\frac{a g}{r}, \\
\frac{1}{r} \frac{d a}{d r}=\frac{g\left(g^{2}-1\right)}{f} .
\end{gathered}
$$

In addition, as we demonstrate below, the resulting generalizing function $f(r)$ is not necessarily finite at the boundaries.

The first analytical model has the profile $g(r)$ defined by

$$
g(r)=\frac{r}{\sqrt[4]{1+r^{4}}}
$$

from which one gets, according to (43), the corresponding gauge solution

$$
a(r)=\frac{1}{1+r^{4}}
$$

whilst (44) gives the following generalizing function:

$$
f(r)=\frac{\left(1+r^{4}\right)^{5 / 4}\left(\sqrt{1+r^{4}}-r^{2}\right)}{4 r} .
$$

It has the following behavior at the boundaries: $f(0)=$ $f(\infty)=\infty$. However, even in this case, one still achieves well-behaved solutions possessing finite total energy. To clarify the way it happens, we calculate the fields $B(r)$ and $H(r)$ arising from (46) and (45). The magnetic field

$$
B(r)=\frac{4 r^{2}}{\left(1+r^{4}\right)^{2}}
$$

presents a ring-like profile, a typical magnetic behavior related to the $|\phi|^{6}$-vortices; see the dotted blue line in Figure 3. The auxiliary function $H(r)$ reads as

$$
H(r)=-\frac{\left(\sqrt{1+r^{4}}-r^{2}\right)^{2}}{4 \sqrt{1+r^{4}}},
$$

which, at the boundaries, assumes the values $H(0)=-1 / 4$ and $H(\infty)=0$, as desired. As a result, the analytical profiles (45) and (46) give rise to a BPS vortex whose total energy is $E_{\mathrm{bps}}=\pi / 2$.

The second $|\phi|^{6}$-model is a little bit more sophisticated than the previous ones, with the Higgs profile being given by

$$
g(r)=e^{-(1 / 4) \operatorname{Ei}\left(1,(1 / 4) r^{4}\right)},
$$

with the function $\mathrm{Ei}(1, r)$ standing for the exponential integral

$$
\operatorname{Ei}(1, r) \equiv \int_{1}^{\infty} \frac{e^{-r x}}{x} d x
$$

Nevertheless, the gauge field has a simpler solution,

$$
a(r)=e^{-(1 / 4) r^{4}}
$$

whereas the corresponding functions $f(r)$ and $H(r)$ are defined by

$$
\begin{gathered}
f(r)=-\frac{e^{-(1 / 2) \operatorname{Ei}\left(1,(1 / 4) r^{4}\right)}-1}{r^{2}} e^{-(1 / 4) \operatorname{Ei}\left(1,(1 / 4) r^{4}\right)+(1 / 4) r^{4}}, \\
H(r)=-\frac{\left(e^{-(1 / 2) \operatorname{Ei}\left(1,(1 / 4) r^{4}\right)}-1\right)^{2}}{r^{2}} e^{-(1 / 2) \operatorname{Ei}\left(1,(1 / 4) r^{4}\right)},
\end{gathered}
$$

from which we get the fact that $f(0)=\infty$ and $f(\infty)=$ 0 , whilst $H(\infty)=0$ and $H(0)=-(1 / 2) \sqrt{e^{\gamma}}, \gamma$ being Euler's constant $(\gamma=0.5772156649 \ldots)$. We see that $f(r)$ is divergent at the origin, whilst vanishing asymptotically. Notwithstanding, as in the previous case, the Bogomol'nyi bound for the BPS total energy saturates at $E_{\mathrm{bps}}=\pi \sqrt{e^{\gamma}}$. The magnetic field of the resulting self-dual configuration,

$$
B(r)=r^{2} e^{-(1 / 4) r^{4}},
$$


also presents the aforecited typical ring-like behavior; see the long-dashed gold line in Figure 3.

In the sequel, we depict all the analytical solutions together with the standard ANO profile, from which we highlight their main features and also the differences of the generalized solutions in comparison with the usual MaxwellHiggs ones.

The analytical solutions defining the Higgs profiles $g(r)$ are depicted in Figure 1. The profile (32) is plotted with the dash-dotted red line, whilst (37) is represented by the dashed green line; these solutions correspond to the generalized $|\phi|^{4}$ models. On the other hand, the dotted blue line stands for (45) and the long-dashed gold line represents (50), both belonging to the noncanonical $|\phi|^{6}$-models. The standard (numerical) $n=1$ ANO Higgs profile is drawn with the solid black line. The overall conclusion is that the analytical solutions behave in the same general way the standard one does. However, the new profiles saturate the asymptotic value $g(r=\infty)=1$ faster, so that the new Higgs profiles are more localized, and the corresponding bosons are more massive than the ANO ones. From now on, we follow the same line/color definitions established in Figure 1.

The gauge profiles $a(r)$ are plotted in Figure 2. There, we see that the profiles (45) and (50), related to the $|\phi|^{6}$ potential, have developed a plateau close to the origin, with such structure being a common feature presented in the selfdual $|\phi|^{6}$-scenarios. On the other hand, the profiles (33) and (38), corresponding to the $|\phi|^{4}$-models, are lumps centered at the origin. The amplitude in $r=0$ corresponds to the winding number $n=1$, as already commented. For large radius, all profiles vanish monotonically.

We show the profiles we have found for the magnetic field $B(r)$ in Figure 3. The solutions regarding the fourth-order potential are lumps centered at the origin, just as the ANO magnetic field. On the other hand, the solutions related to the sixth-order potential present a ring-like behavior, as expected when considering the magnetic fields belonging to the selfdual $|\phi|^{6}$-vortices. We also observe differences both on the amplitudes and on the characteristic lengths of the magnetic profiles.

At last, the BPS energy densities are depicted in Figure 4. We see that all the profiles are lumps centered at origin, as expected for $n=1$ vortices (including the $|\phi|^{6}$-ones). Besides the different characteristic lengths, it is also interesting to note that the profiles coming from the fourth-order potential have achieved greater amplitudes than those ones related to the sixth-order potential.

In the next section, we present our final considerations and the perspectives regarding future works.

\section{Ending Comments}

We have investigated the existence of analytical BPS vortices within the nonstandard Maxwell-Higgs scenario proposed in [46], with such model being generalized by two positive functions, namely, $f(|\phi|)$ and $w(|\phi|)$, which change the overall dynamics of the original theory; see (1). By imposing the radially symmetric ansatz (12) for the gauge and scalar fields, we have reviewed the BPS framework and achieved the first-order equations whose corresponding solutions have finite energy. The self-duality arises when the condition (26) is satisfied by the potential $U$ and the functions $f$ and $w$.

The existence of a well-defined Bogomol'nyi bound (19) is ruled by an auxiliary function $H(r)$ obeying appropriated boundary conditions which guarantee a finite total energy. So, the well-behaved $H(r)$ selects the function $f(r)$ defining the generalized model. With this prescription, we have obtained analytical profiles to some $n=1$ self-dual vortex configurations within both $|\phi|^{4}$ - and $|\phi|^{6}$-models saturating different energy bounds.

In order to compare the analytical self-dual solutions with the ANO ones, we have depicted the profiles for the scalar, gauge, and magnetic fields, in Figures 1, 2, and 3, respectively, whilst the BPS energy density is plotted in Figure 4 . The overall conclusion is that the new profiles are well-behaved in all relevant sectors, assuring the consistence of the generalized models here proposed. Furthermore, all the solutions have provided localized energy densities and magnetic fields, as expected. In general, they mimic the behavior of the well-known numerical configurations, as the ring-like magnetic field related to the self-dual $|\phi|^{6}$-vortices.

Concerning the possibility of obtaining analytical solutions with higher winding numbers, no obvious route for it seems to be available within the models we have studied in this work. Nevertheless, it is possible to construct analytical BPS vortices possessing higher vorticity but different generalizing functions $f(r)$. Such procedure is clarified by the following example related to the $|\phi|^{6}$-models. We propose

$$
g(r)=\frac{r^{n}}{\left(1+r^{m}\right)^{n / m}},
$$

with $m, n>0$, as a generalization for the Higgs profile given by (45). This leads to

$$
a(r)=\frac{n}{1+r^{m}},
$$

compatible with the boundary conditions for a vortex possessing any integer winding number (i.e., $n=+1,+2,+3, \ldots$ ). The resulting magnetic field is

$$
B(r)=\frac{m n r^{m-2}}{\left(1+r^{m}\right)^{2}},
$$

whilst the corresponding $f(r)$ and $H(r)$ are

$$
\begin{aligned}
f(r) & =\frac{r^{n-m+2}}{n m} R^{2-3 n / m}\left(R^{2 n / m}-r^{2 n}\right)^{2}, \\
H(r) & =\frac{r^{2 n-m+2}}{m} R^{1-6 n / m}\left(R^{2 n / m}-r^{2 n}\right)^{2},
\end{aligned}
$$

respectively, with $R=1+r^{m}$. However, only the case $m=$ $2 n+2$ provides positive $f$ and $w$, nonsingular magnetic field and finite energy. Obviously, the choice $n=1$ or $m=4$ reduces this general proposal to the case (45). The generalization of the vortex configurations presenting higher 
winding numbers within other field scenarios can follow this general idea.

Regarding future works, interesting issues include the search for the nontopological self-dual vortices arising in the generalized Maxwell-Higgs scenario (1) when endowed by the sixth-order potential (42); see [47]. In parallel, some of us are also working in a general formulation of the deformation method [48-50] applicable to field models possessing generalized dynamics [51]. These two fronts are now under investigation, and we expect interesting results for a future report.

\section{Conflict of Interests}

The authors declare that there is no conflict of interests regarding the publication of this paper.

\section{Acknowledgment}

The Brazilian authors thank CAPES, CNPq, and FAPEMA for partial financial support.

\section{References}

[1] N. Manton and P. Sutcliffe, Topological Solitons, Cambridge University Press, Cambridge, UK, 2004.

[2] E. Bogomol'nyi, “The stability of classical solutions," Soviet Journal of Nuclear Physics, vol. 24, pp. 449-454, 1976.

[3] M. Prasad and C. Sommerfield, "Exact classical solution for the 't Hooft Monopole and the Julia-Zee Dyon," Physical Review Letters, vol. 35, no. 12, p. 760, 1975.

[4] D. Finkelstein, "Kinks," Journal of Mathematical Physics, vol. 7, pp. 1218-1225, 1966.

[5] A. A. Abrikosov, "On the magnetic properties of superconductors of the second group," Soviet Physics JETP, vol. 5, pp. 1174$1182,1957$.

[6] H. B. Nielsen and P. Olesen, "Vortex-line models for dual strings," Nuclear Physics B, vol. 61, pp. 45-61, 1973.

[7] G. Hooft, "Magnetic monopoles in unified gauge theories," Nuclear Physics B, vol. 79, no. 2, pp. 276-284, 1974.

[8] A. M. Polyakov, "Particle spectrum in quantum field theory," JETP Letters, vol. 20, no. 6, pp. 194-195, 1974.

[9] D. Bazeia, E. da Hora, C. dos Santos, and R. Menezes, "Generalized self-dual Chern-Simons vortices," Physical Review D, vol. 81, Article ID 125014, 2010.

[10] D. Bazeia, E. da Hora, R. Menezes, H. P. de Oliveira, and C. dos Santos, "Compactlike kinks and vortices in generalized models," Physical Review D: Particles, Fields, Gravitation and Cosmology, vol. 81, no. 12, Article ID 125016, 2010.

[11] C. dos Santos and E. da Hora, "Domain walls in a generalized Chern-Simons model," The European Physical Journal C, vol. 70, no. 4, pp. 1145-1151, 2010.

[12] C. dos Santos and E. da Hora, "Lump-like solitons in a generalized Abelian-Higgs Chern-Simons model," The European Physical Journal C, vol. 71, article 1519, 2011.

[13] C. dos Santos, "Compact solitons in an Abelian-Higgs ChernSimons model," Physical Review D, vol. 82, Article ID 125009, 2010.
[14] D. Bazeia, E. da Hora, and D. Rubiera-Garcia, "Compact vortexlike solutions in a generalized Born-Infeld model," Physical Review D, vol. 84, no. 12, Article ID 125005, 2011.

[15] C. dos Santos and D. Rubiera-Garcia, "Generalized sineGordon solitons," Journal of Physics A, vol. 44, no. 42, Article ID 425402, 2011.

[16] D. Bazeia, R. Casana, E. da Hora, and R. Menezes, "Generalized self-dual Maxwell-Chern-Simons-Higgs model," Physical Review D-Particles, Fields, Gravitation and Cosmology, vol. 85, no. 12, Article ID 125028, 2012.

[17] C. Adam, L. A. Ferreira, E. da Hora, A. Wereszczynskiand, and W. J. Zakrzewski, "Some aspects of self-duality and generalised BPS theories," Journal of High Energy Physics, vol. 2013, article 62, 2013.

[18] E. Babichev, "Global topological $k$-defects," Physical Review D, vol. 74, no. 8, Article ID 085004, 2006.

[19] C. Adam, N. Grandi, J. Sanchez-Guillen, and A. Wereszczyński, "K fields, compactons and thick branes," Journal of Physics A: Mathematical and Theoretical, vol. 41, Article ID 212004, 2008.

[20] C. Adam, J. Sanchez-Guillen, and A. Wereszczynski, " $k$-defects as compactons," Journal of Physics A, vol. 40, no. 45, pp. 1362513643, 2007.

[21] C. Adam, J. Sánchez-Guillén, and A. Wereszczyński, "BPS Skyrme model and baryons at large $N_{c}$," Physical Review D, vol. 82, Article ID 085015, 2010.

[22] C. Adam, N. Grandi, P. Klimas, J. Sánchez-Guillén, and A. Wereszczyński, "Compact self-gravitating solutions of quartic $(K)$ fields in brane cosmology," Journal of Physics A: Mathematical and Theoretical, vol. 41, no. 37, Article ID 375401, 2008.

[23] C. Adam, P. Klimas, J. Sánchez-Guillén, and A. Wereszczyński, "Compact gauge K vortices," Journal of Physics A: Mathematical and Theoretical, vol. 42, no. 13, Article ID 135401, 19 pages, 2009.

[24] M. Andrews, M. Lewandowski, M. Trodden, and D. Wesley, "Distinguishing $k$-defects from their canonical twins," Physical Review D, vol. 82, Article ID 105006, 2010.

[25] C. Adam, J. M. Queiruga, J. Sanchez-Guillen, and A. Wereszczynski, " $N=1$ supersymmetric extension of the baby Skyrme model," Physical Review D, vol. 84, Article ID 025008, 2011.

[26] C. Adam, J. M. Queiruga, J. Sanchez-Guillen, and A. Wereszczynski, "Supersymmetric $k$ field theories and defect structures," Physical Review D, vol. 84, Article ID 065032, 2011.

[27] P. P. Avelino, D. Bazeia, R. Menezes, and J. G. G. S. Ramos, "Localized D-dimensional global k-defects," The European Physical Journal C, vol. 71, article 1683, 2011.

[28] D. Bazeia, J. D. Dantas, A. R. Gomes, L. Losano, and R. Menezes, "Twinlike models in scalar field theories," Physical Review D, vol. 84, Article ID 045010, 2011.

[29] A. Mostafazadehm, "Imaginary-scaling versus indefinitemetric quantization of the Pais-Uhlenbeck oscillator," Physical Review D, vol. 84, Article ID 105018, 2011.

[30] C. Adam and J. M. Queiruga, "Algebraic construction of twinlike models," Physical Review D, vol. 84, Article ID 105028, 2011.

[31] C. Adam and J. M. Queiruga, “Twinlike models with identical linear fluctuation spectra," Physical Review D, vol. 85, Article ID 025019, 2012.

[32] C. Adam, C. Naya, J. Sanchez-Guillen, and A. Wereszczynski, "Vector BPS Skyrme model," Physical Review D, vol. 86, no. 8, Article ID 085001, 2012. 
[33] C. Adam, C. Naya, J. Sanchez-Guillen, and A. Wereszczynski, "Vector BPS baby Skyrme model," Physical Review D, vol. 86, Article ID 045015, 2012.

[34] D. Bazeia, E. da Hora, and R. Menezes, "Twinlike models for self-dual Maxwell-Higgs theories," Physical Review D, vol. 85, Article ID 045005, 2012.

[35] D. Bazeia, A. S. Lobao Jr., and R. Menezes, “Twinlike models for kinks and compactons in flat and warped spacetime," Physical Review D, vol. 86, Article ID 125021, 2012.

[36] C. Armendáriz-Picón, T. Damour, and V. Mukhanov, " $k$ inflation," Physics Letters B, vol. 458, no. 2-3, pp. 209-218, 1999.

[37] E. Babichev, V. Mukhanov, and A. Vikman, "k-essence, superluminal propagation, causality and emergent geometry," Journal of High Energy Physics, vol. 2008, no. 2, article 101, 2008.

[38] V. Mukhanov and A. Vikman, "Enhancing the tensor-to-scalar ratio in simple inflation," Journal of Cosmology and Astroparticle Physics, no. 02, p. 004, 2005.

[39] A. Sen, “Tachyon matter," Journal of High Energy Physics, vol. 7, article 065, 2002

[40] C. Armendariz-Picon and E. A. Lim, "Haloes of $k$-essence," Journal of Cosmology and Astroparticle Physics, vol. 2005, no. 08, p. 007, 2005.

[41] J. Garriga and V. F. Mukhanov, "Perturbations in $k$-inflation," Physics Letters B, vol. 458, no. 2-3, pp. 219-225, 1999.

[42] R. J. Scherrer, "Purely kinetic $k$-essence as unified dark matter," Physical Review Letters, vol. 93, Article ID 011301, 2004.

[43] A. D. Rendall, "Dynamics of $k$-essence," Classical and Quantum Gravity, vol. 23, no. 5, pp. 1557-1569, 2006.

[44] R. Casana, M. M. Ferreira, E. da Hora, and C. dos Santos, "Analytical self-dual solutions in a nonstandard Yang-MillsHiggs scenario," Physics Letters B, vol. 722, no. 1-3, pp. 193-197, 2013.

[45] R. Casana, M. M. Ferreira Jr., and E. da Hora, "Generalized BPS magnetic monopoles," Physical Review D, vol. 86, Article ID 085034, 2012.

[46] D. Bazeia, E. da Hora, C. dos Santos, and R. Menezes, "BPS solutions to a generalized Maxwell-Higgs model," The European Physical Journal C, vol. 71, no. 12, pp. 1-9, 2011.

[47] D. Bazeia, R. Casana, M. M. Ferreira Jr., and E. da Hora, Submitted to The European Physical Journal C.

[48] D. Bazeia, L. Losano, and J. M. C. Malbouisson, "Deformed defects," Physical Review D, vol. 66, no. 10, Article ID 101701, 2002.

[49] C. A. Almeida, D. Bazeia, L. Losano, and J. M. C. Malbouisson, "New results for deformed defects," Physical Review D, vol. 69, no. 6, Article ID 067702, 2004.

[50] L. Losano, J. M. C. Malbouisson, D. Rubiera-Garcia, and C. dos Santos, "Deformation method for generalized Abelian HiggsChern-Simons models," Europhysics Letters, vol. 101, no. 3, Article ID 31001, 2013.

[51] D. Bazeia, R. Casana, M. M. Ferreira Jr., E. da Hora, and L. Losano, work in preparation. 

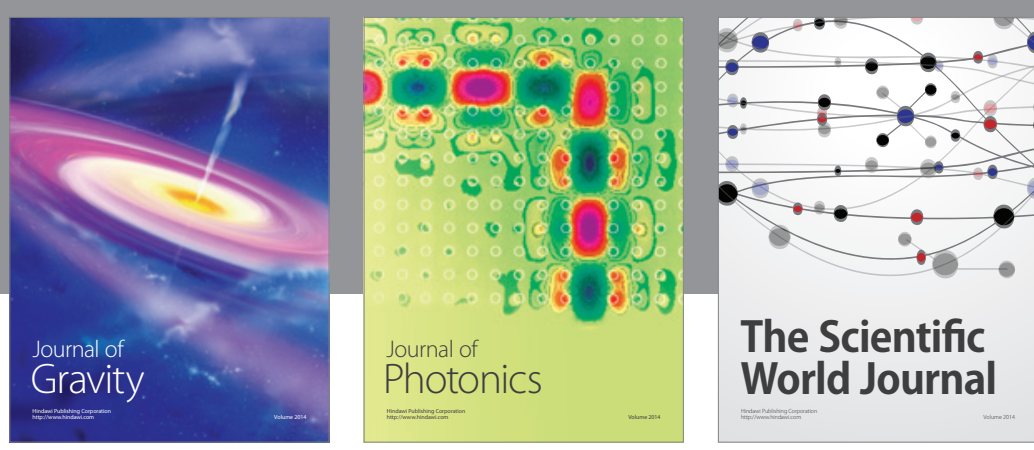

The Scientific World Journal
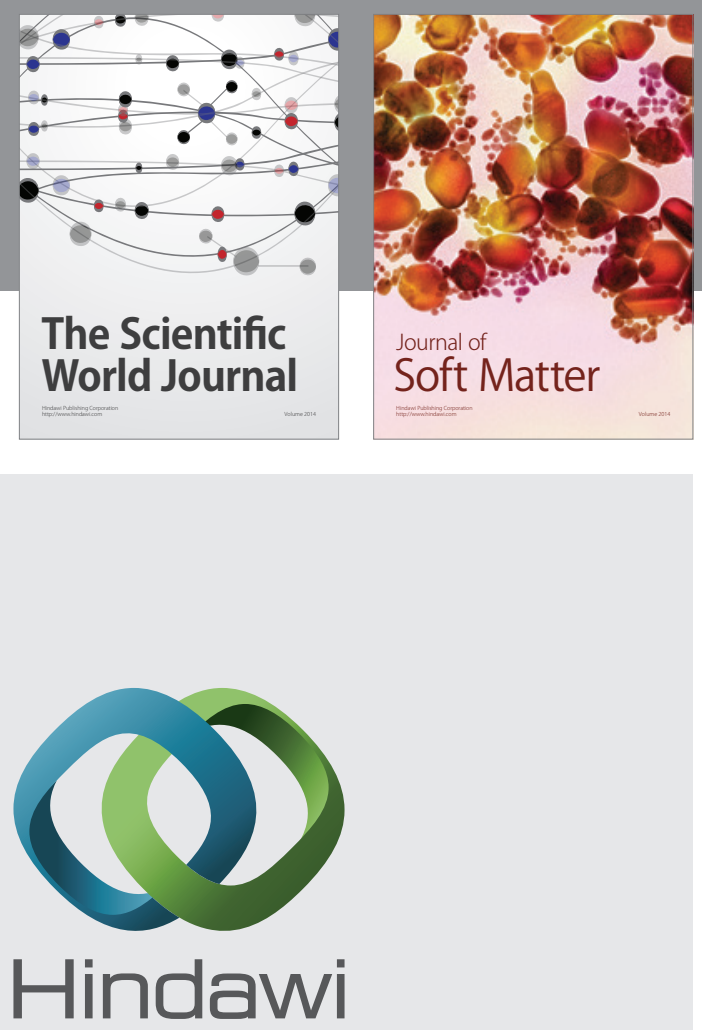

Submit your manuscripts at

http://www.hindawi.com

nternational Journal of

Statistical Mechanics
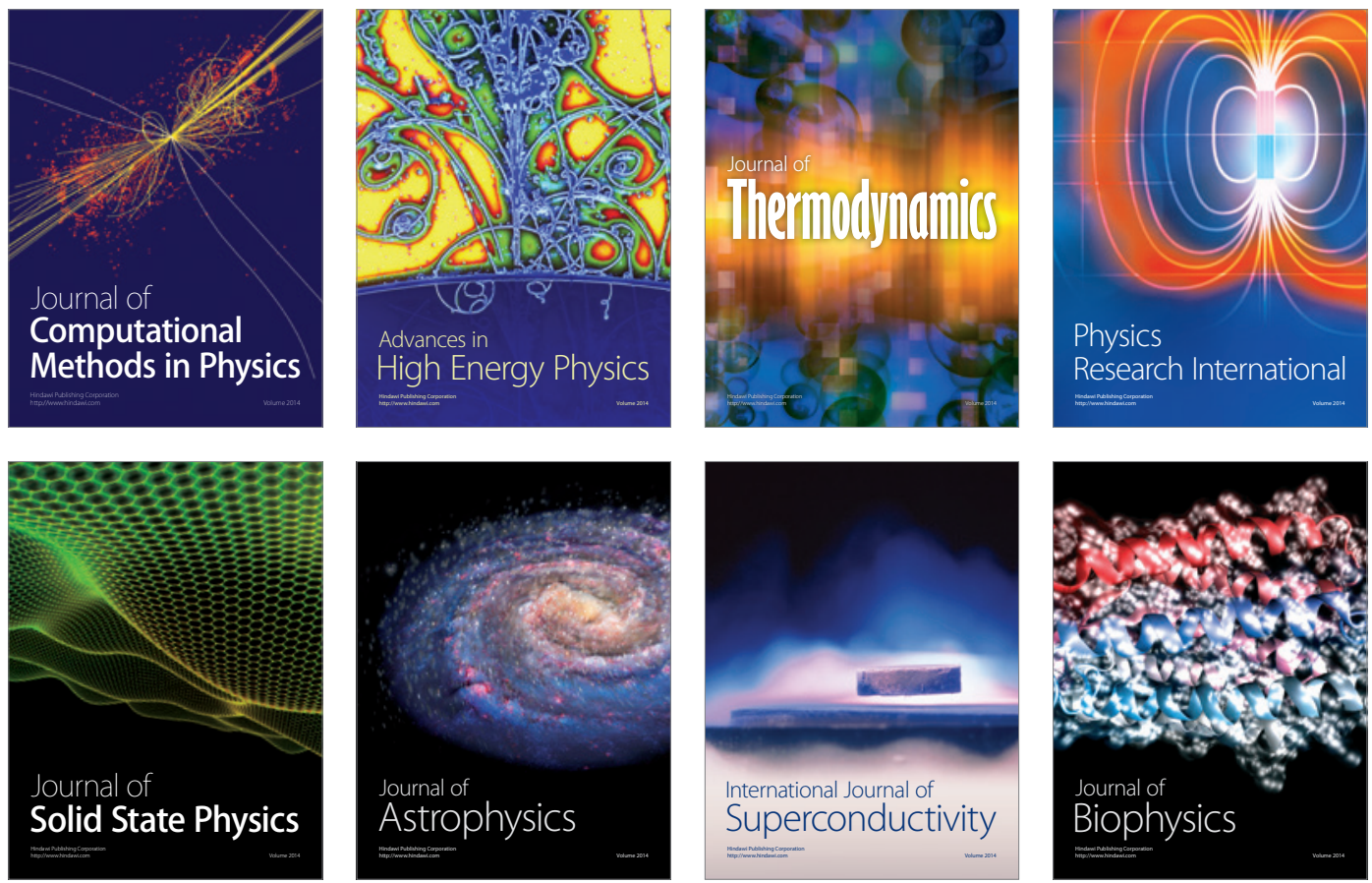
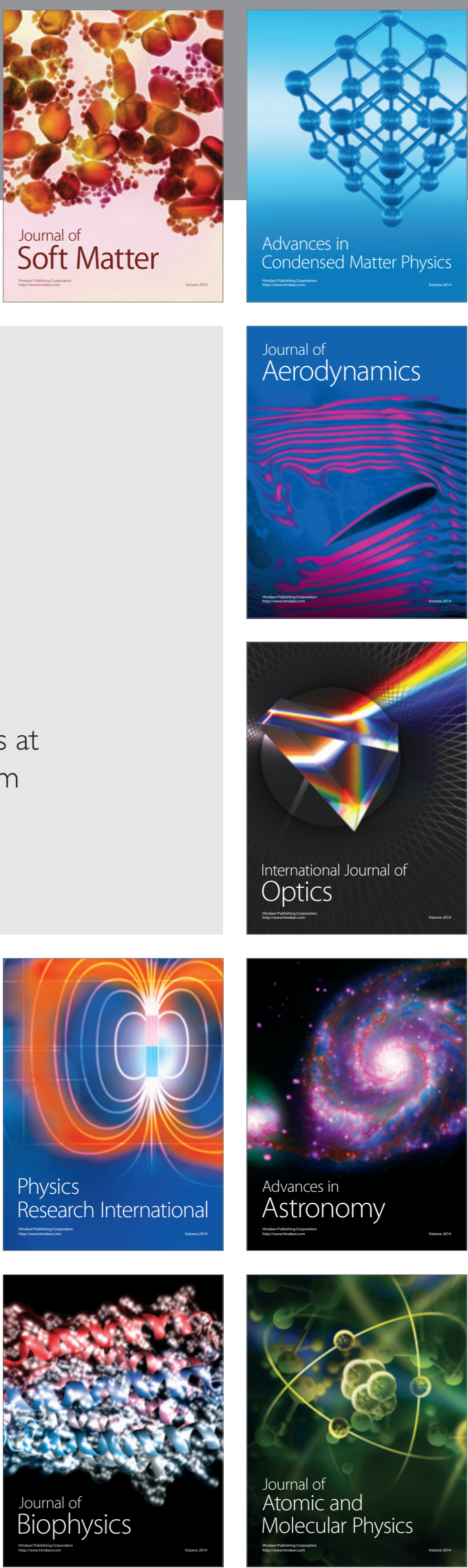